of ERA in a population of EIA patients from an Early Arthritis Research Center (EARC).

Methods: We have assessed all patients with EIA referred to our EARC between 2012-2016. Patients who were diagnosed with other diseases except EIA or ERA, or in whom symptom duration exceeded 12 months, were excluded from the analysis. Every patient underwent clinical, laboratory and ultrasound evaluation. For the proposed US diagnostic method we have evaluated bilaterally 3 joints: wrists, MCP II and III and 2 tendon regions: the extensor ulnaris carpis tendon and the flexor tendons of the fingers. The presence/absence of synovitis/tenosynovitis either in gray-scale or power-Doppler scale was scored in a binary mode as 1/0. In order to simplify the scanning protocol, we considered the flexor tendons of the fingers as a singular structure for each hand which meant that that the presence of US abnormalities in at least one flexor tendon was scored as 1 . Thus, the maximum score obtainable in both hands was 10 . We analyzed the performance of the proposed US method for the diagnosis of ERA using ROC curve analysis. Results: Of 253 patients referred to our EARC, 73 satisfied the inclusion criteria; among them, 43 fulfilled the EULAR/ACR criteria for RA (ERA patients), while the other 30 were considered to have undifferentiated EIA. The demographic, clinical and US data of the 73 patients are displayed in the table below. 34/43 ERA patients $(76.7 \%)$ had a duration of symptoms less or equal to 3 months which classifies them as very ERA (VERA).

Table 1. Demographic, clinical, laboratory and US data of the study patients - data are either $n$ (\%), mean \pm SD or median (IQR)

\begin{tabular}{lccc}
\hline Parameters & EIA $(\mathrm{n}=30)$ & ERA $(\mathrm{n}=43)$ & $\mathrm{p}$ \\
\hline Gender (Female) & $17(56.7 \%)$ & $27(62.8 \%)$ & 0.816 \\
Age & $41.70 \pm 15.58$ & $55.47 \pm 13.71$ & $<0.001$ \\
Mean duration of symptoms & $3.16 \pm 3.22$ & $3.54 \pm 3.58$ & 0.556 \\
CRP $(\mathrm{mg} / \mathrm{l})$ & $7.85(1.99-26.20)$ & $18.62(3.57-14.68)$ & 0.848 \\
ESR $(\mathrm{mm} / \mathrm{h})$ & $26.00(10.00-44.75)$ & $34.53(14-51)$ & 0.238 \\
RF $(\mathrm{IU} / \mathrm{ml})$ & $10.00(7.85-14.56)$ & $142.75(35.14-201.74)$ & $<0.001$ \\
ACPA $(\mathrm{IU} / \mathrm{ml})$ & $5.00(0.5-5.00)$ & $153.96(46.20-212.00)$ & $<0.001$ \\
DAS28 & $4.00(3.35-5.05)$ & $4.89(4.31-5.60)$ & $<0.001$ \\
SDAl & $17.82(12.28-26.37)$ & $27.96(20.92-34.61)$ & $<0.001$ \\
US evaluation & $0(0-3)$ & $5(3-7)$ & $<0.001$ \\
\hline
\end{tabular}

In ROC analysis, a cut-off of the US score of 4 had best results for sensitivity and specificity $(73.3 \%$ and $82.1 \%$, respectively), with an area under the curve of 0.812 . The US score correlated with the levels of RF, ACPA, DAS28 and SDAI $(p<0.001)$, but not with those of acute phase reactants $(p>0.05)$. The time needed for performing the ultrasound examination was less than 10 minutes.

Conclusions: The proposed US method proves to be reliable in identifying patients with ERA. The binary mode of US evaluation allows even persons with little training in US examination to diagnose patients. As the costs and time needed for US evaluation are low, the method is valuable in clinical practice for a rapid assessment of patients with EIA.

References:

[1] Aletaha D et al. Arhritis Rheum. 2010;62:2569-258.

Disclosure of Interest: None declared

DOI: 10.1136/annrheumdis-2017-eular.4604

\section{FRI0677 ROLE OF NAILFOLD VIDEOCAPILLAROSCOPY AND 22-MHZ DOPPLER ULTRASOUND IN THE ASSESSMENT OF SYSTEMIC SCLEROSIS-RELATED DIGITAL VASCULOPATHY}

T. Schioppo $^{1,2}$, A. Orenti ${ }^{1,3}$, P. Boracchi ${ }^{1,3}$, O. De Lucia $^{2}$, A. Murgo $^{2}$,

P.L. Meroni ${ }^{1,2}, \mathrm{~F}$. Ingegnoli ${ }^{1,2}$ on behalf of OPERA study group. ${ }^{1}$ Università degli Studi di Milano; ${ }^{2}$ ASST Pini-CTO; ${ }^{3}$ Lab of Medical Statistics, Epidemiology and Biometry GA Maccacaro, Milan, Italy

Background: Microvascular damage plays a critical role in the initiation and perpetuation of systemic sclerosis (SSc). A comprehensive approach should investigate both superficial and deep layers of peripheral microcirculation. In addition to nailfold videocapillaroscopy (NVC), a well-established technique to evaluate outer skin layer vessels, power Doppler ultrasound (PDUS) has been recently used to study microcirculation in the inner levels [1].

Objectives: To study the severity of microvascular involvement in patients with SSc by using both NVC to measure capillary density (outer layer at the nailfold area) and PDUS to detect perfusion (deeper layers at the nailfold and pulp area). Methods: 100 SSc consecutive patients fulfilling the 2013 EULAR classification criteria were enrolled. PDUS was performed at the 3rd and 4th finger of the dominant hand after exclusion of ulnar artery occlusion (UAO). In case of UAO non-dominant hand was examined. Ultrasound investigation was performed with Esaote MyLab 70 XVG by means of linear array transducer (10-22 MHz). Power Doppler settings were standardized (Doppler frequency $14.3 \mathrm{MHz}$, Gain $55 \%$, PRF $750 \mathrm{~Hz}$ ). PDUS measurements included sagittal scan of nailbed and fingertip qualitatively graded from 1 (no signal) to 4 (marked hyperemia) [2], and resistivity index (RI) of ulnar and radial proper digital arteries. Capillary density (number/mm) was calculated by NVC with magnification 200X performed on two images of the same digits examined by PDUS.

Results: 100 SSc patients, 87 (87\%) women, 86 (86\%) limited cutaneous SSc, median age 62.2 years old, median disease duration 8 years were evaluated. 7 (7\%) patients had UAO. Concordance between fingertip and nailbed perfusion as assessed by PDUS is reported in Table 1.

\begin{tabular}{lcccccc}
\hline Table 1 & \multicolumn{6}{c}{ Nailbed PDUS } \\
\cline { 3 - 7 } & & Grade 1 & Grade 2 & Grade 3 & Grade 4 \\
\hline Fingertip PDUS & Grade 1 & 15 & 19 & 3 & 1 & 38 \\
& Grade 2 & 13 & 13 & 6 & 6 & 38 \\
& Grade 3 & 3 & 5 & 10 & 10 & 28 \\
& Grade 4 & 2 & 8 & 15 & 71 & 96 \\
& Sum & 33 & 45 & 34 & 88 & 200 \\
\hline
\end{tabular}

Concordance between fingertip and nailbed perfusion as assessed by PDUS is equal to 0.7398 . The lower $97.5 \%$ confidence interval limit is 0.6433 .

Association between capillary density, and fingertip and nailbed perfusion as assessed by PDUS is shown in Table 2 .

Table 2

\begin{tabular}{lcc}
\hline & Cabillary density & $\begin{array}{c}\text { p-value of the difference between the mean } \\
\text { of the category, with respect to reference (grade 1) }\end{array}$ \\
\hline $\begin{array}{c}\text { Fingertip PDUS } \\
\text { Grade 1 }\end{array}$ & \\
Grade 2 & 2.895 & 0.038 \\
Grade 3 & 3.763 & 0.181 \\
Grade 4 & 3.500 & 0.007 \\
Nailbed PDUS & 3.844 & \\
Grade 1 & 3.212 & \\
Grade 2 & 3.433 & 0.597 \\
Grade 3 & 3.294 & 0.854 \\
Grade 4 & 3.949 & 0.049 \\
\hline
\end{tabular}

Conclusions: To our knowledge, this is the first study to correlate NVC and PDUS finding in SSc patients. Fingertip and nailbed PDUS grade concordance was found to be satisfactory. The mean capillary density tends to be greater with respect to grade 1 . This is particularly evident comparing grade 4 and grade 1 . As such, these two imaging techniques provide different and potentially complementary information on SSc-related peripheral microvascular involvement. There is potential clinical utility in these observations that has yet to be unlocked fully.

References:

[1] Lescoat A et al. Arthritis Care Res. 2016;Epub ahead of print.

[2] Newman JS et al. Radiology. 1996,198:582-584.

Disclosure of Interest: None declared

DOI: 10.1136/annrheumdis-2017-eular.3859

\section{FRI0678 ULTRASOUND-GUIDED SYNOVIAL NEEDLE BIOPSY: SINGLE CENTER EXPERIENCE OF AN EMERGING, MINIMALLY INVASIVE TECHNIQUE IN CLINICAL PRACTICE AND RESEARCH}

V.C. Romao ${ }^{1,2}$, J. Polido-Pereira ${ }^{1,2}$, R. Barros ${ }^{1}$, E. Vieira-Sousa ${ }^{1,2}$, R. Luís ${ }^{3}$, E. Vitorino ${ }^{3}$, F. Saraiva ${ }^{1}$, J.E. Fonseca ${ }^{1,2},{ }^{1}$ Rheumatology Department, Hospital de Santa Maria, CHLN; ${ }^{2}$ Rheumatology Research Unit, Instituto de Medicina Molecular, Faculty of Medicine, University of Lisbon; ${ }^{3}$ Pathology Department, Hospital de Santa Maria, CHLN, Lisbon, Portugal

Background: Synovial biopsy remains an important tool in clinical practice and research for the study of synovitis. Ultrasound-guided needle biopsy (USNB) has recently emerged as a minimally invasive technique, which enables collection of high quality synovial tissue with very good patient tolerance.

Objectives: To report the experience with USNB in our department, since its introduction in late 2013.

Methods: We reviewed the clinical files of all patients who had an USNB in our department. Degree of US joint synovitis was evaluated on a semi-quantitative scale (0-3) in terms of synovial thickness (ST) and power Doppler (PD). Since 2015, we assessed patient tolerance and acceptance of the procedure using a standardized questionnaire, which includes visual analogue scales (VAS) of pain, stiffness and swelling of the biopsied joint. Changes in US and VAS scores were assessed using the Wilcoxon signed-rank test.

Results: Forty-eight patients had 53 USNB, mostly for diagnostic purposes $(79 \%)$, performed by 4 different operators - Figure 1. All types of joints were biopsied, mostly medium sized (26 wrists, 7 ankles), but also large (3 knees, 4 shoulders, 6 elbows, 3 hips) and small (1 sternoclavicular, 1 naviculocuneiform, 1 metacarpophalangeal and 1 proximal interphalangeal) joints, 2 bursae (subacromial) and 1 tendon sheath. USNB was repeated in the same joint (wrist) twice in 3 patients and three times in one patient. Procedures were well tolerated, with $67 \%$ of patients classifying it as easy or very easy, $78 \%$ reporting no or only mild discomfort and $77 \%$ considering likely/very likely to accept to repeat the biopsy. An increase in analgesic medication in the days following the biopsy was reported by 13 out of 44 questioned patients. After a median of 8 days following the procedure, a significant decrease was observed in VAS scores of pain, stiffness and swelling of the biopsied joint, although $23 \%$, $23 \%$ and $31 \%$ of the patients reported small increases in these scores (median $9.5,11$ and $10 \mathrm{~mm}$, respectively). There was no significant change in US scores pre- and post-biopsy, with only 3 and 2 patients having an increase in ST or PD scores, respectively. Biopsies were overall safe, with 6 minor immediate adverse events $(11 \%)$. There were no cases of haemartrosis, joint/periarticular 